Technical Communication

\title{
Validation study of the distinct lattice spring model (DLSM) on P-wave propagation across multiple parallel joints
}

\author{
J.B. Zhu ${ }^{\mathrm{a}, *}$, G.F. Zhao ${ }^{\mathrm{b}, \mathrm{c}}$, X.B. Zhao ${ }^{\mathrm{d}}$, J. Zhao ${ }^{\mathrm{a}}$ \\ a Ecole Polytechnique Fédérale de Lausanne (EPFL), Laboratory for Rock Mechanics (LMR), EPFL-ENAC-IIC-LMR, Station 18, CH-1015 Lausanne, Switzerland \\ ${ }^{\mathrm{b}}$ School of Civil and Environmental Engineering, The University of New South Wales, Sydney, NSW 2052, Australia \\ ' State Key Laboratory for Geomechanics and Deep Underground Engineering, China University of Mining and Technology, Xuzhou, Jiangsu 221116, China \\ ${ }^{\mathrm{d}}$ NJU-ECE Institute for Underground Space and Geo-environment, School of Earth Sciences and Engineering, Nanjing University, Nanjing 210093, China
}

\section{A R T I C L E I N F O}

\section{Article history:}

Received 16 August 2010

Received in revised form 10 December 2010

Accepted 10 December 2010

Available online $\mathrm{xxxx}$

\section{Keywords:}

Distinct lattice spring model

UDEC

Virtual wave source

Wave propagation

Rock joint

\begin{abstract}
A B S T R A C T
A validation study of the distinct lattice spring model (DLSM) for wave propagation problems is performed. DLSM is a microstructure-based numerical model, which is meshless and has advantages in modelling dynamic problems where stress wave propagation is important. To verify the applicability of DLSM to modelling wave propagation through a discontinuous medium, the virtual wave source (VWS) method is used to obtain analytical solutions for wave propagation across a jointed rock mass. Numerical modelling results of the commercial code UDEC are selected as the reference. The effects of particle size and lattice rotation angle on wave propagation are first studied. Then, the results of wave transmission across a single joint with a different joint stiffness and across multiple parallel joints with different joint spacings are derived with DLSM, UDEC and VWS. These results are in good agreement with each other. Therefore, the capability of DLSM to model P-wave propagation across jointed rock mass is verified, which provides confidence for the further application of DLSM to modelling more complex problems.
\end{abstract}

(c) 2010 Elsevier Ltd. All rights reserved.

\section{Introduction}

Joints can significantly influence wave propagation through a rock mass. Analytical and experimental studies on wave propagation across one joint set have been extensively studied [1-4], by focusing on the effects of joint stiffness, incident angle, number of joints and joint spacing on wave propagation. Recently, combined with the equivalent medium model, Li et al. [5] introduced a new concept of virtual wave source (VWS) to study wave propagation across a joint set, where multiple wave reflections among joints were taken into account.

Compared with theoretical and experimental studies, numerical modelling provides a convenient, economical approach to study wave propagation across a jointed rock mass, especially for complicated cases where theoretical solutions are impossible to obtain and experiments are difficult to conduct. The representation of joints is a key difficulty in numerical modelling. In the finite element method (FEM), joints are often treated as individual elements called joint elements [6,7]. Boundary interfaces are often used to model joints with the FEM and boundary element method (BEM) [8] or between BEMs $[9,10]$. Joints are treated as slide lines in the finite difference method (FDM) [11]. These treatments are applica-

\footnotetext{
* Corresponding author. Tel.: +41 21693 5387; fax: +41216934153.

E-mail address: jianbo.zhu@epfl.ch (J.B. Zhu).
}

ble only when the number of joints and their displacement are small. In the discrete element method (DEM), a rock mass is represented as an assembly of discrete blocks and joints as interfaces between the blocks [12]. The universal distinct element code (UDEC), which is a 2D DEM-based numerical program, has been widely adopted to study wave propagation across jointed rock masses. Lemos [13] performed a study on S-wave attenuation across a single joint with Coulomb slip behaviour using UDEC. Brady et al. [14] performed UDEC modelling on the slip of a single joint under an explosive line source. Chen [15] verified the capability of UDEC to model the responses of jointed rock masses under explosion loading. Zhao et al. [16] carried out numerical studies of P-wave propagation across multiple non-linearly deformable joints with UDEC.

In the present study, a newly developed numerical code, the distinct lattice spring model (DLSM), was used to study normally incident wave propagation across a joint set. DLSM is a microstructure-based numerical model. Compared with traditional numerical methods, DLSM has the following advantages in modelling wave propagation problems:

(1) discontinuities are easy to implement for both the weak material layer and virtual joint plane method;

(2) the computational model is easy to generate due to the advantage of the meshless properties of DLSM; 
(3) DLSM has potential to model continuum-discontinuum wave propagation problems, e.g., wave induced damage and the influence of fracturing on the wave propagation process.

The DLSM can be viewed as a meshless method like EFG and FPM, but with similarities to DEM. Compared with the particle based DEM, the DLSM can directly use macroscopic parameters without calibration process, which is regarded as the main advantage over other discrete element based methods. Moreover, DLSM also has advantages over existing meshless methods, e.g., EFG, FPM and $\mathrm{SPH}$, on stability, integration requirement and convenience to deal with heterogeneity problems. Due to the meshless and natural discrete properties of DLSM, it is suitable for dynamic fracturing simulation.

In this paper, the virtual wave source, which is originated but different from the method used by Li et al. [5], was used to obtain the analytical solutions. The commercial code UDEC was used to obtain the reference numerical solutions. The objective of the paper is to compare the results of DLSM with corresponding results from UDEC and analytical solutions and therefore to verify the applicability of DLSM in modelling wave propagation across jointed rock masses.

\section{The distinct lattice spring model (DLSM)}

\subsection{The model}

The DLSM $[17,18]$ is a microstructure-based numerical model based on the Realistic Multidimensional Inter Bond (RMIB) model [19], which is an extension of the Virtual Multidimensional Inter Bond (VMIB) model [20]. In DLSM, materials are discretised into mass particles linked through distributed bonds (see Fig. 1a). Whenever two particles are detected in contact, they are linked together through bonds between their centre points. Due to the explicit considerations of the material microstructure, the model has the potential to generate more realistic modelling of material

(a)
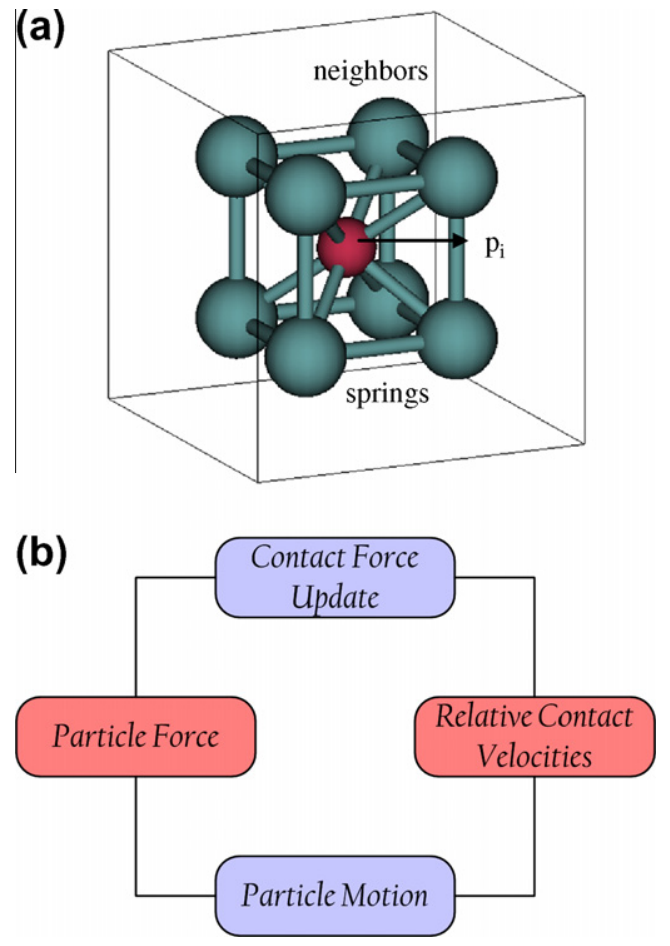

Fig. 1. (a) Physical model and (b) calculation cycle of DLSM. failure behaviour than a phenomenological model does. Based on Cauchy-Born rules and the hyper-elastic theory, the relationship between the micromechanical parameters and the macro material constants can be obtained as follows [21]:

$k_{n}=\frac{3 E}{\alpha^{3 D}(1-2 v)}$

$k_{s}=\frac{3(1-4 v) E}{\alpha^{3 D}(1+v)(1-2 v)}$

where $k_{n}$ is the spring normal stiffness, $k_{s}$ is the shear stiffness, $E$ is the Young's modulus, $v$ is the Poisson ratio and $\alpha^{3 D}$ is the microstructure geometry coefficient, which is obtained from

$\alpha^{3 D}=\frac{\sum l_{i}^{2}}{V}$

where $l_{i}$ is the original length of the $i t h$ bond and $V$ is the volume of the geometry model.

The particles and springs comprise a whole system, which represents the material. For this system, the equation of motion is expressed as

$[\mathbf{K}] \mathbf{u}+[\mathbf{C}] \dot{\mathbf{u}}+[\mathbf{M}] \ddot{\mathbf{u}}=\mathbf{F}(\mathbf{t})$

where $u$ represents the particle displacement vector, $[\mathbf{M}]$ is the diagonal mass matrix, $[\mathbf{C}]$ is the damping matrix and $\mathbf{F}(t)$ is the vector of external forces on particles. The motion equations of the particle system are solved through an explicit central finite differences scheme. The calculation cycle is illustrated in Fig. 1b. The details of the implementation and verification of DLSM can be found in $[17,18]$.

\subsection{Representation of discontinuities in DLSM}

The idea of the virtual joint plane method [21] is to represent joints in DLSM. The work principle of the smooth-joint contact model is shown in Fig. 2, where the relative displacement increment between the two particles is decomposed into components normal and tangential to the smooth joint surface. Then the force-displacement law is applied in the smooth joint coordinates.

When a spring is cut by the virtual joint plane, its spring parameters are modified according to following rules:

(a) changing the direction of the original spring into the normal vector of the virtual joint plane,

(b) replacing the spring stiffnesses with

$k_{n}^{\text {bond }}=\frac{k_{n}^{j} A l^{*}}{n^{\text {cut }}}$

$k_{s}^{\text {bond }}=\frac{k_{s}^{j} A l^{*}}{2 n^{\text {cut }}}$

where $k_{n}^{\text {bond }}$ and $k_{s}^{\text {bond }}$ are the normal and shear stiffnesses of the bond, $k_{n}^{j}$ and $k_{s}^{j}$ are the inputted joint stiffness parameters of the discontinuity, A is the area of the joint plane, $l^{*}$ is the mapped bond length on the joint plane normal direction and $n^{\text {cut }}$ is the number of bonds cut by the joint plane.

\subsection{Non-reflection boundary condition in DLSM}

The finite boundary of the computational model causes elastic waves to be reflected and mixed with the original wave, which makes analysis of the modelling results more difficult. To solve these problems, an artificial boundary condition that can simulate a computational model without any finite boundaries is needed. This kind of boundary condition is also called a non-reflection 


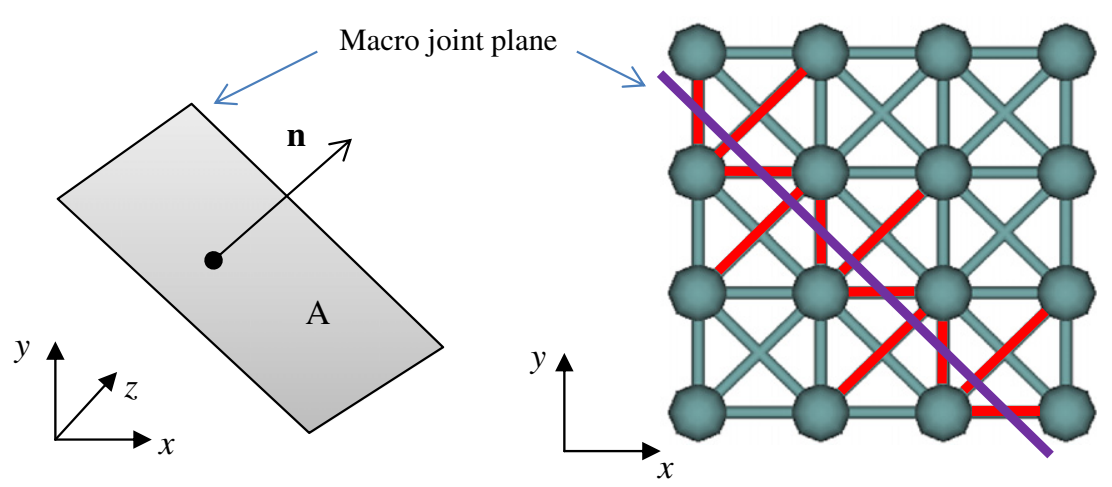

Fig. 2. Virtual joint plane method in DLSM to represent discontinuity.

boundary condition, which can eliminate the spurious reflections induced by the finite boundary. A number of non-reflection boundary conditions have been proposed in past years. The viscous element method proposed by Lysmer and Kuhlemeyer [22] is the oldest and simplest artificial boundary condition and is implemented in DLSM in this paper. Fig. 3 illustrates the imposition of a viscous element-based non-reflection boundary condition in DLSM. Three dashpots are applied to particles of the artificial boundary plane. The reflected wave is minimised by imposing these damping dashpots at the finite boundary. The mechanical properties of these dashpots can be determined through the material properties of the linked particle. The normal and shear viscous tractions can be written as

$t_{n}=-\rho \bar{A} C_{p} v_{n}$

$t_{s 2}=-\rho \bar{A} C_{s} v_{s 1}, t_{s 2}=-\rho \bar{A} C_{s} v_{s 2}$

where $\rho$ is the material density of the linked particle, $\bar{A}$ is the equivalent area given as $\sqrt[3]{V}, V$ is the volume of the particle, $v_{n}$ are the normal components of the particle velocity, $v_{s 1}$ and $v_{s 2}$ are the shear components of the velocity and $C_{p}$ and $C_{s}$ are the P-wave and Swave velocities, which are given by

$C_{p}=\sqrt{\frac{K+4 G / 3}{\rho}}, C_{s}=\sqrt{\frac{G}{\rho}}$

where $K$ and $G$ are the bulk and shear elastic modulus of the linked particle. The non-reflection boundary is implemented in DLSM by including Eqs. (7) and (8) in the force calculation procedure of DLSM (shown in Fig. 1b).
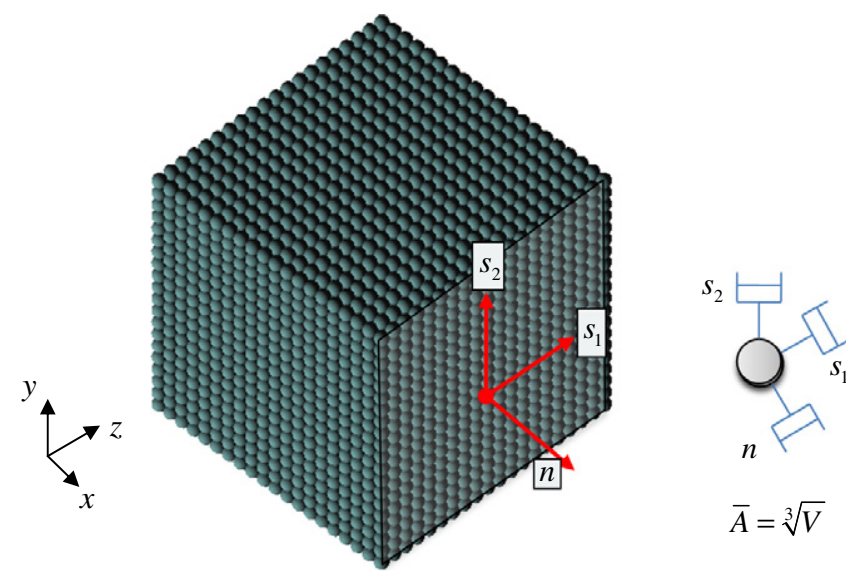

Fig. 3. Implementation of viscous non-reflection boundary condition in DLSM.

\section{The virtual wave source (VWS) method}

To verify DLSM, VWS was used to obtain analytical solutions for wave propagation across a joint set. Li et al. [5] introduced the concept of virtual wave source (VWS) to study wave propagation across a joint set, where multiple wave reflections among joints were considered. When the wave arrives at the joint position, a reflected wave is "created" by VWS, while the transmitted wave is derived by using the effective viscoelastic moduli of the rock mass. The concept of VWS used in the present study is based on but different from that of Li et al. [5]. Combined with the displacement discontinuity model, VWS exists at the joint position and represents the mechanical properties of the joint. It produces one reflected wave and one transmitted wave each time the incident wave arrives at the VWS position.

To obtain the theoretical solution of the transient wave transmitted across a joint set, the incident transient wave is first transformed into the frequency domain by fast Fourier transform (FFT). In the frequency domain, the incident transient wave can be transformed as the sum of a series of harmonic waves with different amplitudes and frequencies. The reflected and transmitted waves upon a single joint can be obtained by the reflection and transmission coefficients [23]. When there are multiple joints, the transmitted harmonic wave across a joint set is the result of the wave superposition of different transmitted waves produced by the VWS. Then an inverse transform for these transmitted harmonic waves is conducted to obtain the transmitted transient wave by inverse fast Fourier transform (IFFT), which can transform one series of harmonic waves into a transient wave.

\section{Validation of DLSM}

\subsection{Influence of particle size on wave propagation}

The influence of particle size on the numerical accuracy of DLSM in modelling wave propagation must be studied first. Similar studies have been performed for some mesh based methods $[11,15]$. The mesh ratio (lr), which is defined as the ratio of the mesh size to the incident wavelength, is used as the controlling parameter. The same term is used in DLSM to maintain consistency with previous studies, but it is defined as the ratio of the diameter of the particle to the incident wavelength. In the following section, the influence of $l r$ on the numerical precision of DLSM in modelling wave propagation is investigated. The main objective is to obtain an appropriate value of $l r$.

A plane elastic P-wave propagating through a continuous, homogeneous, isotropic and elastic medium can be taken as the ideal example for verifying the numerical accuracy of DLSM. The DLSM models used are shown in Fig. 4, where the particle diameter 


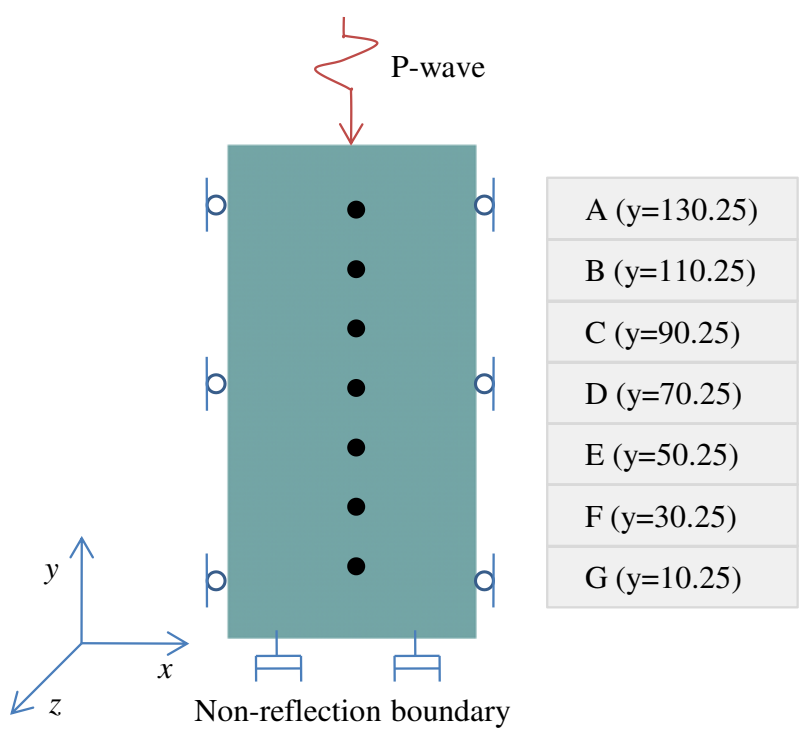

Fig. 4. DLSM model for P-wave propagation.

is equal to $0.5 \mathrm{~mm}$. Seven measuring points were positioned in the specimen to record the time histories of the P-wave. The basic properties of the material are as follows: the rock density is $2120 \mathrm{~kg} / \mathrm{m}^{3}$, the Young's modulus is $27.878 \mathrm{GPa}$, and the Poisson's ratio is 0.2987 . A one-cycle sinusoidal wave with unit amplitude was normally applied to the top boundary and propagated along the $y$ direction through the model. The left and right side boundaries were fixed in the $x$-direction when modelling P-wave propagation through the DLSM model. The frequencies of the P-wave varied among $0.1 \mathrm{MHz}, 0.2 \mathrm{MHz}, 0.5 \mathrm{MHz}, 1.0 \mathrm{MHz}$ and $2.0 \mathrm{MHz}$ to model different $l r$. The relation between $l r$ and the average percentage error is shown in Fig. 5. The percentage error decreased with decreasing $l r$. To obtain sufficiently accurate results, a magnitude of $\operatorname{lr}$ equal to 1/420 was adopted in the following study.

\subsection{Influence of lattice rotation angle on wave propagation}

In order to check the anisotropic behaviour of the DLSM model, the original model is rotated to different angles $\left(0^{\circ}, 15^{\circ}, 30^{\circ}, 45^{\circ}\right)$ as shown in Fig. 6. It should be noted that the model is periodic for every $45^{\circ}$. The magnitude of transmission coefficient across a single joint $\left|T_{1}\right|$ is calculated with the rotated models and the original model, as shown in Table 1. In the calculation, the joint stiffness is

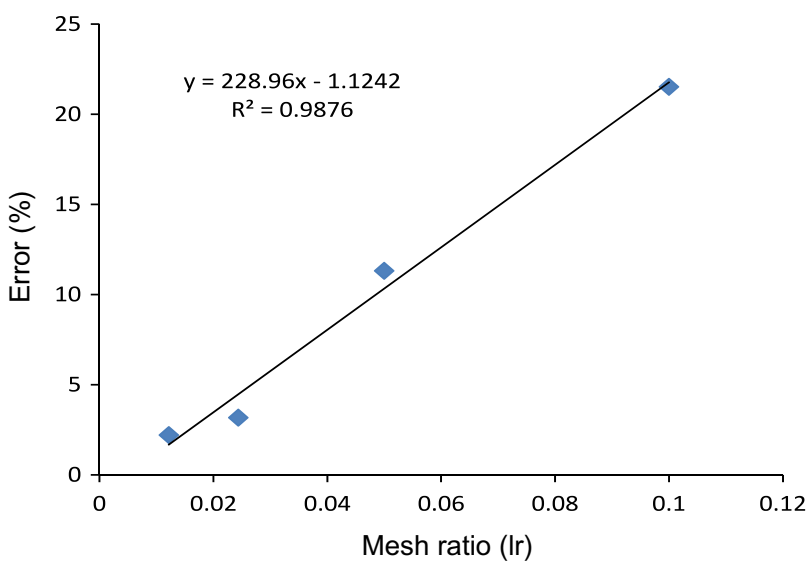

Fig. 5. Relationship between average percentage error and $l r$ for DLSM in modelling P-wave propagation.
$400 \mathrm{GPa}$, and the measurement points are the same for all the models. From the results shown in Table 1, it can be seen that the differences caused by the rotation are small. The maximum errors occur when rotational angles are equal to $15^{\circ}$ and $30^{\circ}$. However, when the rotational angle is equal to $45^{\circ}$, the calculated result is almost the same as that with the original model $\left(0^{\circ}\right)$. It can be explained through the smoothness and symmetry of the models. When the lattice rotation angles are equal to $0^{\circ}$ or $45^{\circ}$, the boundaries of the model are smooth, and the left and right boundaries of the model are symmetric. While the rotation angles are equal to $15^{\circ}$ or $30^{\circ}$, the boundaries are not smooth, and the left and right boundaries are not symmetric. The calculated results will be more accurate when the boundaries of the model are smooth and symmetric.

\subsection{Wave propagation across a single joint}

Results of wave propagation across a single joint by DLSM are compared with those by UDEC and analytical solutions by VWS.

UDEC was the first discrete element program developed by Cundall [24] to study fractured rock masses. In UDEC, blocks represent rock materials, which can be rigid or deformable; contacts represent joints, which are deformable. A force-displacement relation is used at the contacts, and contact forces are subsequently obtained from joint displacements (closure, opening and slip) calculated from block movements. The contact forces and displacements are continuously found in a series of calculations, which trace block movements and disturbance propagation caused by applied loads and body forces. This process is dynamic, and the disturbance propagation speed depends on the physical properties of the discrete system.

The DLSM model was the same as that shown in Fig. 4, except that there was one joint, which was assumed to be dry and elastic. The properties of the rock material were the same as those adopted in Section 4.1. The incident P-wave was a half-cycle sinusoidal wave with a frequency of $20 \mathrm{kHz}$ and unit amplitude. Therefore, Ir was equal to $1 / 420$. The model used in UDEC was the same as that of DLSM, where $l r$ is equal to $1 / 24$ [15]. Fig. 7 shows $\left|T_{1}\right|$ as a function of normalised joint normal stiffness $K_{n}$, which is equal to $k_{n} / \omega Z$, where $k_{n}$ is the joint normal stiffness, $\omega$ is the angular frequency and $Z$ is the acoustic impedance. Fig. 8 shows the transmitted waveforms across a single joint with DLSM and analytical solutions for $k_{n}=2000 \mathrm{GPa}$. It can be found from Figs. 7 and 8 that:

(1) $\left|T_{1}\right|$ from DLSM agrees well with those from UDEC and analytical solutions. The waveform across a single joint obtained with DLSM is almost the same as that from the analytical solution. Therefore, DLSM is applicable to study wave propagation across a single joint;

(2) $\left|T_{1}\right|$ increases with increasing $K_{n}$. When $K_{n}$ approaches zero, $\left|T_{1}\right|$ also approaches zero, which corresponds to the case of free boundary reflection. When $K_{n}$ approaches infinity, $\left|T_{1}\right|$ approaches 1, which corresponds to the case of an intact rock material without joints;

(3) the frequency of the transmitted wave is lower than that of the incident wave. It indicates that the joint acts as a high frequency filter.

\subsection{Wave propagation across a joint set}

When there are multiple parallel joints, i.e., a set of joints, there are multiple reflections among joints, which makes the wave propagation more complicated. The transmitted wave across one joint set is the result of transmitted waves arriving at different times. In this section, a numerical study with DLSM and UDEC and analytical study with VWS were conducted. The model and the properties 
(a)

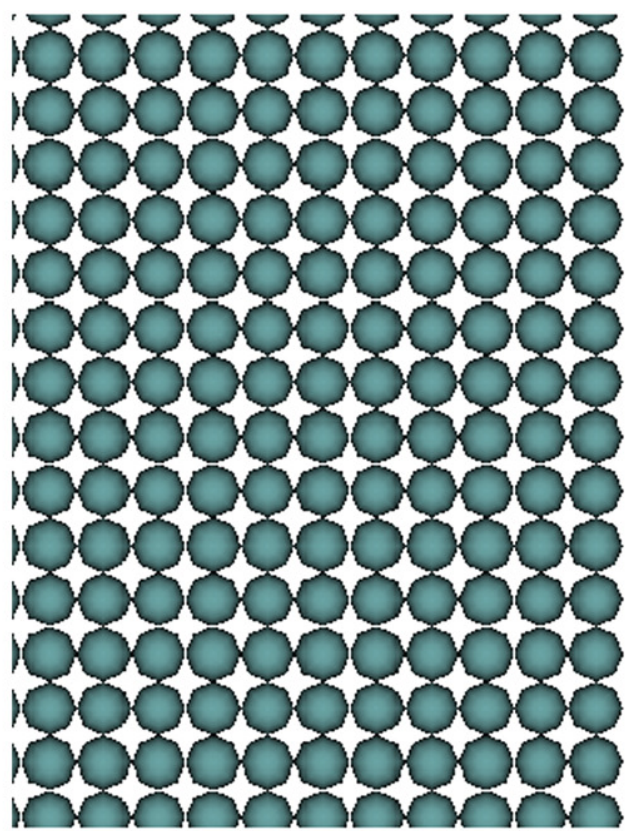

(c)

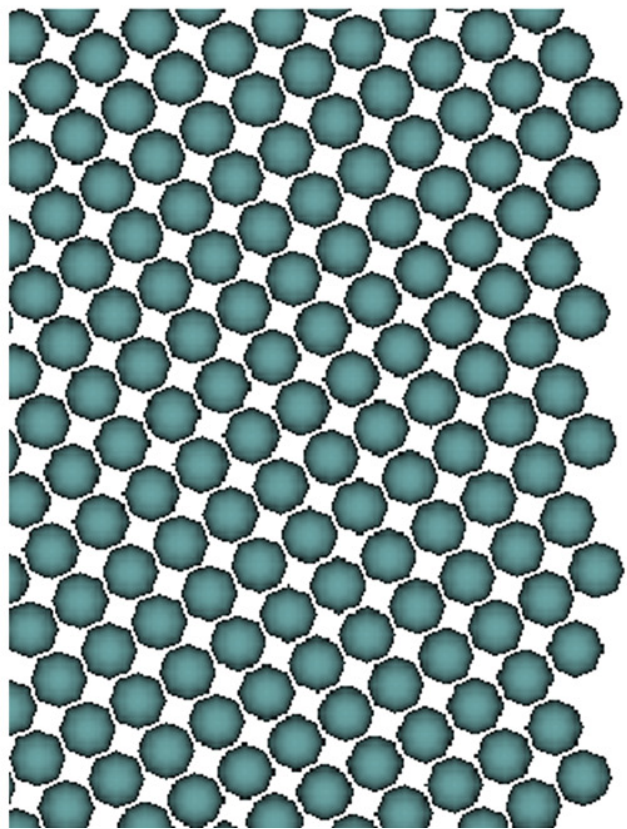

(b)

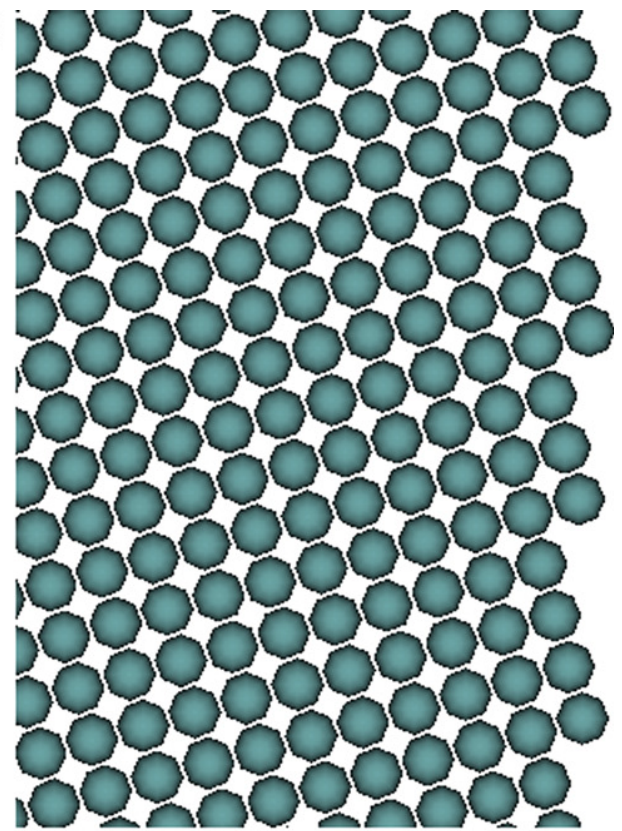

(d)

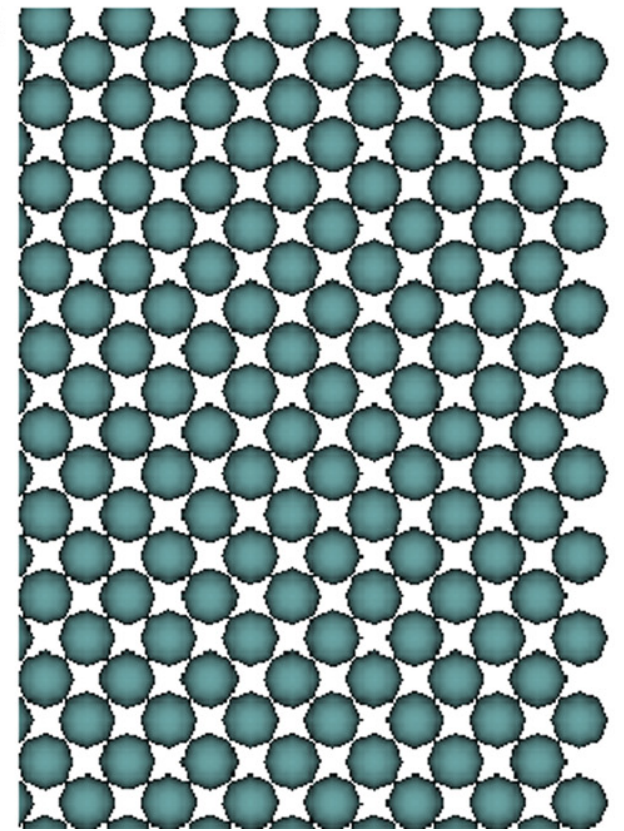

Fig. 6. DLSM models with different lattice rotation angles: (a) $0^{\circ}$, (b) $15^{\circ}$, (c) $30^{\circ}$ and (d) $45^{\circ}$.

Table 1

$\left|T_{1}\right|$ calculated by DLSM with different lattice rotation angles.

\begin{tabular}{lll}
\hline Lattice rotation angle $\left(^{\circ}\right)$ & $\left|T_{1}\right|$ & Error $(\%)$ \\
\hline 0 & 0.6558 & 0 \\
15 & 0.6518 & 0.61 \\
30 & 0.6522 & 0.55 \\
45 & 0.6552 & 0.09
\end{tabular}

of the rock material and the incident wave were the same as those in Section 4.2, except that the length of the model in the $y$ direction was larger in order to input more joints. Fig. 9 shows the magnitude of transmission coefficient across $N$ joints $\left|T_{N}\right|$ for different nondimensional joint spacing $\xi$, which is defined as the ratio of joint spacing to the incident wavelength, where $k_{n}=1000 \mathrm{GPa}$. Fig. 10 shows the transmitted waveforms across 8 joints with

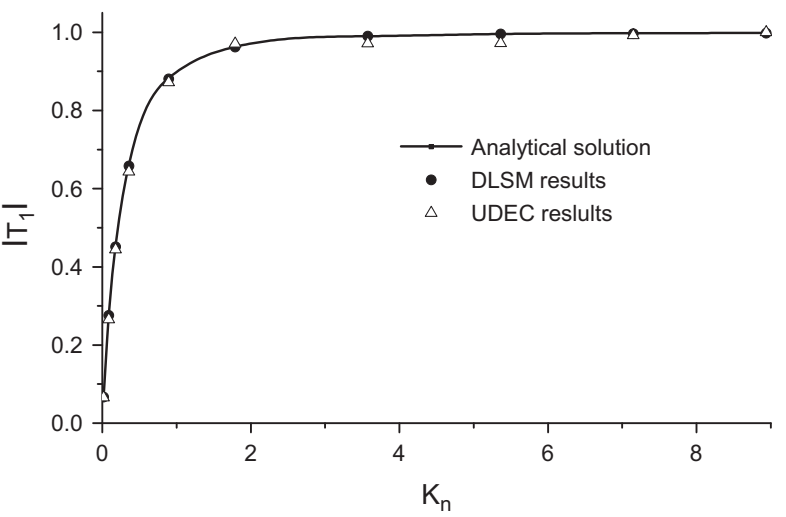

Fig. 7. $\left|T_{1}\right|$ versus $K_{n}=k_{n} / \omega Z$.

Please cite this article in press as: Zhu JB et al. Validation study of the distinct lattice spring model (DLSM) on P-wave propagation across multiple parallel joints. Comput Geotech (2011), doi:10.1016/j.compgeo.2010.12.002 


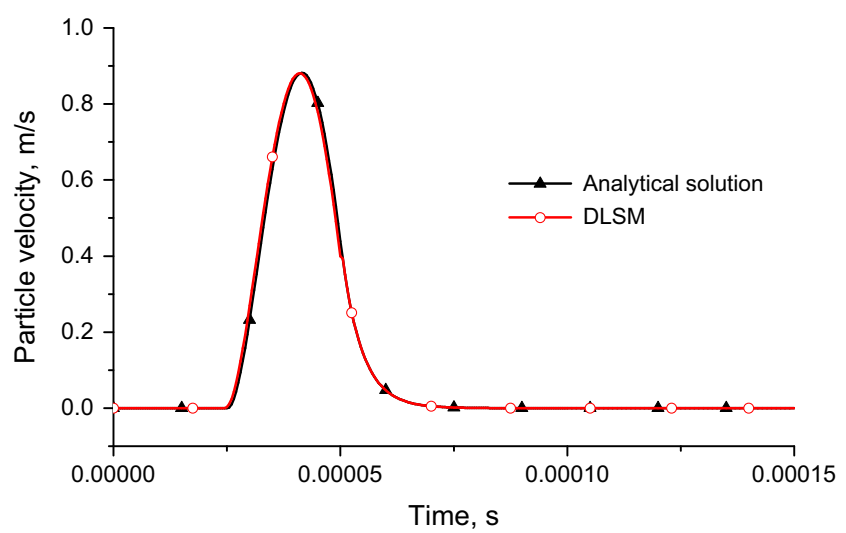

Fig. 8. Transmitted waveforms across a single joint.

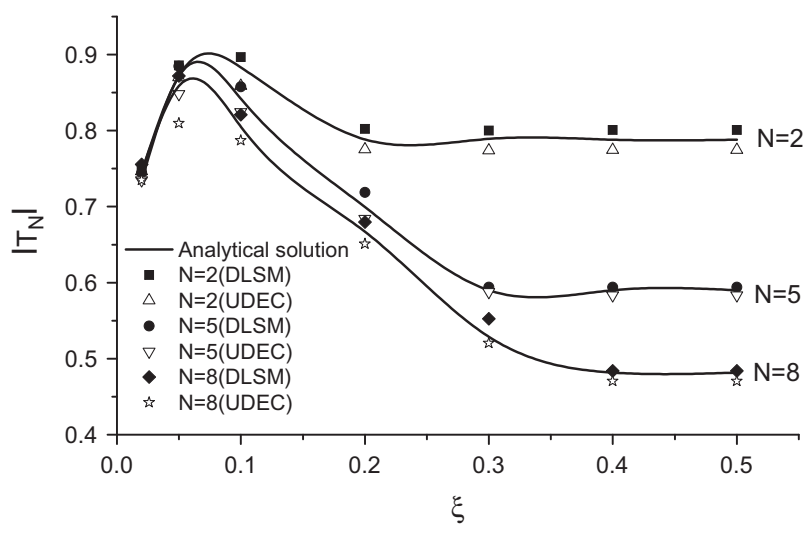

Fig. 9. $\left|T_{N}\right|$ versus $\xi$.

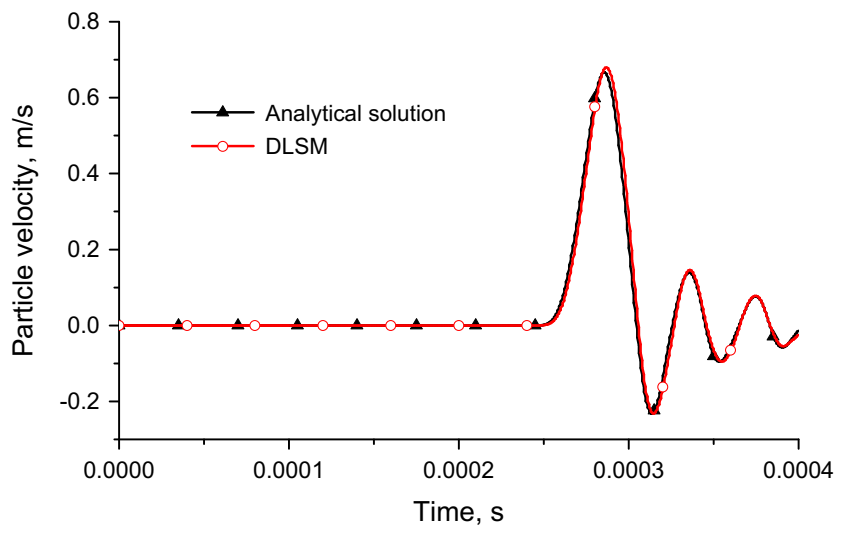

Fig. 10. Transmitted waveforms across 8 joints.

DLSM and analytical solutions for $k_{n}=2000 \mathrm{GPa}$ and $\xi=0.2$. It can be found from Figs. 9 and 10 that:

(1) the transmission coefficients obtained by DLSM agree well with those from UDEC and analytical solutions. Generally speaking, the results obtained by DLSM are slightly larger than those of analytical solutions, while the results from UDEC are a little smaller than analytical solutions. There are only minor differences between the transmitted waveform across 8 joints obtained with DLSM and the analytical solution. Therefore, DLSM is applicable to study wave propagation across a joint set;
(2) with increasing $\xi,\left|T_{N}\right|$ first increases to the maximum value, and then it decreases. When $\xi$ is sufficiently large, $\left|T_{N}\right|$ does not change with $\xi$, which indicates that $\xi$ has no influence on $\left|T_{N}\right|$ because later arriving transmitted waves from interjoint reflections have no effects on the amplitude of the first transmitted wave;

(3) generally speaking, $\left|T_{N}\right|$ decreases with increasing $N$. However, when $\xi$ is small, $\left|T_{N}\right|$ may not change or even increase with increasing $N$.

\section{Conclusions}

The capability of DLSM to model P-wave propagation through multiple rock joints is verified in this paper. To calibrate the numerical results of DLSM, the concept of VWS is adopted to obtain analytical solutions for wave propagation across a joint set, and UDEC is also used to obtain the reference numerical results. The results of DLSM, UDEC and VWS are obtained and compared with each other for wave propagation across a single joint and a set joint. The results of DLSM agree well with those of UDEC and analytical solutions. Therefore, DLSM is proven to be an applicable code to study normally incident P-wave propagation across a discontinuous medium. Further application of the code in more complex conditions, e.g., stress wave propagation induced failure and the influence of dynamic failure on stress wave propagation, needs to be studied.

\section{Acknowledgements}

The authors would like to thank the anonymous reviewer for the valuable comments and helpful suggestions. This research is sponsored by the Swiss National Science Foundation (SNSF) and the China Scholarship Council (CSC).

\section{References}

[1] Kendall K, Tabor D. An ultrasonic study of the area of contact between stationary and sliding surfaces. Proc R Soc London 1971;A323:321-40.

[2] Schoenberg M. Elastic wave behavior across linear slip interfaces. J Acoust Soc Am 1980;68:1516-21.

[3] Pyrak-Nolte LJ, Myer LR, Cook NGW. Anisotropy in seismic velocities and amplitudes from multiple parallel fractures. J Geophys Res 1990;95:11345-58.

[4] Cai JG, Zhao J. Effects of multiple parallel fractures on apparent wave attenuation in rock masses. Int J Rock Mech Min Sci 2000;37:661-82.

[5] Li JC, Ma GW, Zhao J. An equivalent viscoelastic model for rock mass with parallel joints. J Geophys Res 2010. p. 115 doi:10.1029/2008JB006241.

[6] Goodman RE, Taylor RL, Brekke T. A model for the mechanics of jointed rock. J Soil Mech Found Div ASCE 1968;94:637-59.

[7] Ghaboussi J, Wilson EL, Isenberg J. Finite elements for rock joints and interfaces. J Soil Mech Found Div ASCE 1973;99:833-48.

[8] Beer G. Implementation of combined boundary element-finite element analysis with applications in geomechanics. In: Banerjee PK, Watson JO, editors. Developments in boundary element methods. London: Applied Science; 1986. p. 191-226.

[9] Crotty JM, Wardle LJ. Boundary integral analysis of piecewise homogeneous media with structural discontinuities. Int J Rock Mech Min Sci Geomech Abstr 1985;22:419-27.

[10] Pande GN, Beer G, Williams JR. Numerical methods in rock mechanics. New York: Wiley; 1990.

[11] Schwer LE, Lindberg HE. Application brief: a finite element slideline approach for calculating tunnel response in jointed rock. Int J Numer Anal Meth Geomech 1992;16:529-40.

[12] Cundall PA. A computer model for simulating progressive large scale movements in blocky rock systems. In: Proc Symp Int Soc Rock Mech. France: Nancy; 1971. p. 11-8.

[13] Lemos JV. A distinct element model for dynamic analysis of jointed rock with application to dam foundation and fault motion. PhD Thesis. Minneapolis, USA: University of Minnesota; 1987.

[14] Brady BH, Hsiung SH, Chowdhury AH, Philip J. Verification studies on the UDEC computational model of jointed rock. In: Mechanics of jointed and faulted rock. Rotterdam: Balkema; 1990. p. 551-8.

[15] Chen SG. Discrete element modelling of jointed rock mass under dynamic loading, Ph.D. Thesis. Singapore: Nanyang Technological University; 1999.

[16] Zhao XB, Zhao J, Cai JG, Hefny AM. UDEC modelling on wave propagation across fractured rock masses. Comput Geotech 2008;35:97-104. 
[17] Zhao GF, Fang J, Zhao J. A 3D distinct lattice spring model for elasticity and dynamic failure. Int J Numer Anal Meth Geomech 2010 doi:10.1002/nag.930.

[18] Zhao GF, Zhao J. Microscopic numerical modelling of the dynamic strength of brittle rock. In: Ma GW, Zhou YX, editors. Analysis of discontinuous deformation: new developments and applications. Singapore: Research Publishing; 2009. p. 633-40.

[19] Zhao GF, Fang J, Zhao J. A new microstructure-based constitutive model for failure modelling of elastic continuum. Eur J Mech Solid 2009 (submitted).

[20] Zhang ZN, Ge XR. Micromechanical consideration of tensile crack behavior based on virtual internal bond in contrast to cohesive stress. Theor Appl Fract Mech 2005;43:342-59.
[21] Zhao GF Development of micro-macro continuum-discontinuum coupled numerical method. PhD thesis. Ecole Polytechnique Fédérale de Lausanne (EPFL). Switzerland: Lausanne; 2010.

[22] Lysmer J, Kuhlemeyer RL. Finite dynamic model for infinite media. J Eng Mech Div ASCE 1969;95:859-77.

[23] Pyrak-Nolte LJ, Myer LR, Cook NGW. Transmission of seismic waves across single natural fractures. J Geophys Res 1990;95:8617-38.

[24] Cundall PA. UDEC -a generalized distinct element program for modelling jointed rock. Report PCAR-1-80, Peter Cundall Associates, US Army, European Research Office, London, Contract DAJA37-79-C-0548; 1980. 